\title{
Perfil epidemiológico dos pacientes com leishmaniose tegumentar americana no município de Ilhéus - Bahia
}

\section{Epidemiological profile of patients with cutaneous Leishmaniasis in the city of Ilheus - Bahia}

\author{
Soraya Sena Campos ${ }^{1}$, Fleming Sena Campos², Glayciane Costa Gois ${ }^{3}$, Tiago Santos Silva ${ }^{4}$
}

\section{Resumo}

Objetivou-se analisar o perfil epidemiológico de 442 pacientes com leishmaniose tegumentar americana no município de Ilhéus entre os anos de 2007 a 2012. A coleta de dados foi realizada na vigilância epidemiológica do município Ilhéus, utilizando como banco de dados os casos notificados e digitados no sistema nacional de notificação de agravos de leishmaniose tegumentar americana. Foram analisadas informações sobre, sexo, faixa etária, escolaridade, critério de confirmação, tipo da leishmaniose, medicação utilizada, conclusão do caso. No município de Ilhéus a letalidade em leishmaniose tegumentar americana foi nula durante o período de estudo e a partir do ano de maior ocorrência houve um decréscimo dos casos positivos. Os indivíduos mais propícios à doença são do sexo masculino, entre 20 a 49 anos, residente em áreas rurais, pardos, autóctone. A forma mais incidente é a cutânea e a droga mais utilizada para o tratamento é a antimonial pentavalente. Não foi confirmada a sazonalidade da doença entre os meses e anos observados e nenhum surto da doença durante o período estudado no município de Ilhéus - Bahia. As informações deste estudo servirão para auxiliar o planejamento de ações epidemiológicas de saúde pública, visando medidas particulares de proteção conforme caracterização local. A partir destes resultados, espera-se mostrar a importância da notificação de investigação, diagnóstico precoce e tratamento eficaz para reduzir sequelas, bem como contribuir para a prevenção e controle da doença na cidade.

Palavras chave: Diagnóstico. Leishmania. Leishmaniose.

\begin{abstract}
The objective of this study was to analyze the epidemiological profile of 442 patients with American tegumentary leishmaniasis in the municipality of Ilhéus between 2007 and 2012. Data collection was performed in the epidemiological surveillance of Ilhéus municipality, using as a database the cases reported and typed in the national system for notifying American tegumentary leishmaniasis We analyzed information on sex, age, schooling, confirmation criteria, type of leishmaniasis, medication used, conclusion of the case. In the municipality of Ilhéus, lethality in American tegumentary leishmaniasis was zero during the study period and from the year of greatest occurrence there was a decrease in positive cases. The most susceptible individuals are male, between 20 and 49 years old, living in rural, brown, autochthonous areas. The most incident form is cutaneous and the most commonly used drug for treatment is pentavalent antimonial. It was not confirmed the seasonality of the disease between the months and years observed and no outbreak of the disease during the period studied in the municipality of Ilhéus - Bahia. The information in this study will serve to assist the planning of epidemiological actions of public health, aiming at specific measures of protection according to local characterization. From these results, it is expected to show the importance of reporting research, early diagnosis and effective treatment to reduce sequelae, as well as contribute to the prevention and control of disease in the city.
\end{abstract}

Keyword: Diagnosis. Leishmania. Leishmaniasis.

\footnotetext{
${ }^{1}$ Mestre em Saúde Pública, Faculdade de Teologia Fides Reformata; Ilhéus, Bahia, Brasil.

${ }^{2}$ Doutor em Zootecnia, Pós-doutorando pelo Programa de Pós Graduação em Ciência Animal e Pastagens, UFRPE/UAG, Garanhuns - Pernambuco.

${ }^{3}$ Doutora em Zootecnia, Pós-doutoranda pelo Colegiado de Pós Graduação em Ciências Veterinárias no Semiárido, UNIVASF, Petrolina - Pernambuco.

${ }^{4}$ Docente em Zootecnia do Instituto Federal de Educação, Ciência e Tecnologia do Sertão Pernambucano; Ouricuri, Pernambuco, Brasil.
} 


\section{Introdução}

Leishmanioses são antropozoonoses que constituem um grande problema de saúde pública, representando um complexo de doenças com importante espectro e diversidade epidemiológica, é considerada uma das cinco doenças infecto-parasitárias endêmicas de maior relevância mundial (CARDOSO et al., 2015). A Organização Mundial de Saúde (OMS, 2000) estima que 350 milhões de pessoas estejam expostas ao risco de adoecer, com registro aproximado de dois milhões de novos casos e diferentes formas clínicas ao ano (MOTA; MIRANDA, 2011).

A leishmaniose tegumentar americana (LTA) é uma doença causada por protozoários do gênero Leishmania, acomete o homem na pele e/ou na mucosa, principalmente das vias aerodigestivas superiores, é considerada cosmopolita (atinge 88 países) e sua notificação é compulsória em apenas 30 países. A transmissão da doença ocorre pela picada de fêmeas de insetos vetores, conhecidos como flebotomíneos. A maioria das leishmanioses são zoonóticas, os seres humanos são considerados hospedeiros acidentais do parasita quando se expõem ao ciclo de transmissão natural (NOBRES; SOUZA; RODRIGUES; 2013).

No Brasil, o principal padrão de transmissão da LTA é promovido pelo desmatamento, que pode ser explicado pelas modificações que o meio ambiente sofre com a decomposição de árvores abatidas, fazendo com que haja alterações nos locais de abrigo e criadouros dos insetos vetores e, ainda, uma dispersão dos animais silvestres que servem de alimento para as fêmeas hematófagas (GOMES et al., 2015). Com isto, as espécies que conseguem resistir de alguma forma as condições adversas, acabam por explorar novos ambientes e aproximam-se cada vez mais das residências humanas. A evolução constante do número de casos de LTA, neste aspecto, é uma consequência da destruição de florestas, principalmente tropicais, onde vários empreendimentos estão sendo implantados (ALVES; MAGALHÃES; SOUSA, 2012).

As manifestações clínicas variam dependendo da espécie de Leishmania envolvida e da resposta imune do hospedeiro, ocorrendo como infecção subclínica até formas mucosas graves e mutilantes, de evolução crônica. Classifica-se, de acordo com a localização das lesões, em formas cutânea localizada, cutânea disseminada e mucosa (BENTES et al., 2015). É provável que a grande variedade de manifestações clínicas da doença e suas formas de progressão se devam à multiplicidade dos fatores envolvidos na determinação da doença, que incluem o grande número de vetores e reservatórios, além da resistência natural do hospedeiro à infecção e da magnitude da resposta imunológica montada. Também é possível que a diversidade genética de Leishmania se deva à reprodução predominantemente assexual, com a formação de uma população com estrutura clonal, que vem evoluindo separadamente (MOURA, 2013).

O município de Ilhéus está inserido em uma região endêmica de LTA, o litoral sul do Estado da Bahia, dispõe de grande superfície de mata atlântica e possui muitas propriedades produtoras de cacau com reservas florestais bem preservadas, ou seja, contribui como um ambiente propício à produção da LTA. A zona urbana do município também mantém coleções residuais de mata, o que permite um foco de produção e reprodução do vetor favorecendo a transmissão da LTA mesmo nas regiões urbanas, o que denota a importância epidemiológica de se realizar um estudo da doença no contexto baiano e, em particular, em Ilhéus.

Objetivou-se analisar o perfil epidemiológico dos pacientes com Leishmaniose Tegumentar Americana no município de Ilhéus - Bahia no ano de 2007 a 2012.

\section{Material e Métodos}

A pesquisa é um estudo retrospectivo, quantitativo transversal. Realizado através do levantamento de dados de casos registrados de Leishmaniose Tegumentar Americana (LTA) do município de Ilhéus - BA, junto a Secretaria Municipal de Saúde e do Centro de Controle de Zoonoses referente ao período de 2007 a 2012.

Os dados foram obtidos no setor de vigilância epidemiológica do departamento da vigilância à saúde da secretaria de saúde do município de Ilhéus - BA. A cidade de Ilhéus situa-se no estado da Bahia, entre 1447'50” Sul, 3947'50”'oeste, apresentado por 184.231 habitantes (IBGE, 2010), possui um clima tropical úmido com médias pluviométricas anuais entre 2.000 e 2.400 milímetros, com chuvas bem distribuídas ao longo do ano, sendo o verão o período de maior precipitação. Apresenta temperatura média compensada anual em tornos dos $25^{\circ} \mathrm{C}$, tendo, no período de inverno, média de $21^{\circ} \mathrm{C}$, com média das máximas de $26^{\circ} \mathrm{C}$ e a das mínimas de $18^{\circ} \mathrm{C}$. Durante o verão, possui média de $26^{\circ} \mathrm{C}$, com médias máximas de $29^{\circ} \mathrm{C}$ e mínimas de $22^{\circ} \mathrm{C}$ (SANTANA et al., 2014). 
Foram utilizados como banco de dados os casos notificados, investigados, confirmados e digitados no SINAN-NET (Sistema Nacional de Notificação de Agravos), de Leishmaniose Tegumentar Americana (LTA) no período correspondente ao ano de 2007 a 2012. Os dados foram obtidos das fichas de investigação de leishmaniose tegumentar americana, preenchidas nas unidades de saúde do município de Ilhéus e enviadas à vigilância epidemiológica do mesmo município, tendo como fonte de maior notificação a unidade de referência do Centro de Atendimento Especializado (CAE III) que recebe casos de todo o município.

Entre os dados avaliados foram observados a frequência da distribuição dos casos por ano, frequência mensal, sexo, faixa etária, raça, nível de escolaridade, zona de residência, fonte de infecção, doença relacionada ao trabalho, classificação epidemiológica, tipo de entrada, confirmação de casos, forma clínica, lesões, cicatrizes cutâneas, gestantes, intradermorreação de Montenegro (IRM), droga inicial, dose prescrita e evolução do caso e óbitos.

No total, foram obtido 442 fichas de investigação de leishmaniose tegumentar americana. Para tabulação dos dados, foi feito o uso de uma planilha do aplicativo Excel do Pacote Office do Sistema Operacional Windows $10^{\circledR}$ Home Professional ${ }^{\circledR}$, sendo realizados cálculos das percentagens e dos seus respectivos valores absolutos.

\section{Resultados}

Durante o período de estudo, foram notificados e confirmados 442 casos de LTA entre os anos de 20072012. O ano de 2010 foi o que apresentou o maior número de casos, correspondendo a $27,15 \%$ dos casos, e em 2007, foram observados o menor número referente a $5,88 \%$ dos indivíduos infectados (Tabela $1)$.

Para a ocorrência dos casos entre os meses, observou-se que nos meses de fevereiro, março, abril, maio, junho, julho e setembro do ano de 2010 foram os meses que mais ocorreram casos de LTA, perpetuando menor numero de ocorrências no mês de maio dos anos 2007, 2008 e 2009, janeiro de 2010, junho de 2011 e fevereiro e novembro de 2012.

Quanto ao gênero dos pacientes portadores de LTA, $69 \%$ eram do gênero masculino e $31 \%$ eram do gênero feminino. A faixa de maior predominância foi entre $20-34$ e 35 - 49 anos, com 23,54 e 22,62\%, respectivamente, a demais faixa etárias obtiveram a seguintes percentagens: em ano de idade o resultado foi de $1,35 \%$, de $1-9$ anos $7,91 \%$, entre 10 e 19 anos $20,8 \%,>50$ anos $23,54 \%$.

Tabela 1 - Distribuição dos casos de leishmaniose tegumentar americana em indivíduos atendidos nos serviços de saúde do departamento da vigilância à saúde da secretaria de saúde do município de Ilhéus Bahia, nos anos de 2007 - 2012.

\begin{tabular}{|c|c|c|c|c|c|c|}
\hline \multicolumn{7}{|c|}{ Frequência/ano } \\
\hline & 2007 & 2008 & 2009 & 2010 & 2011 & 2012 \\
\hline & 5,88 & 21,04 & 14,71 & 27,15 & 19,00 & 12,22 \\
\hline \multicolumn{7}{|c|}{ Frequência/mês de notificação } \\
\hline & 2007 & 2008 & 2009 & 2010 & 2011 & 2012 \\
\hline Janeiro & 0,90 & 0,68 & 0,45 & 0,45 & 0,68 & 0,68 \\
\hline Fevereiro & 0,68 & 0,90 & 1,36 & 2,71 & 1,81 & 0,00 \\
\hline Março & 0,23 & 1,13 & 1,36 & 4,52 & 2,04 & 0,23 \\
\hline Abril & 0,45 & 0,90 & 1,58 & 3,39 & 2,71 & 0,90 \\
\hline Maio & 0,10 & 0,20 & 0,36 & 0,77 & 0,61 & 0,20 \\
\hline Junho & 0,23 & 1,13 & 0,45 & 1,36 & 0,00 & 0,90 \\
\hline Julho & 0,45 & 1,13 & 1,58 & 2,04 & 2,71 & 2,04 \\
\hline Agosto & 0,23 & 2,71 & 0,90 & 2,26 & 2,49 & 0,90 \\
\hline Setembro & 0,68 & 3,85 & 1,58 & 1,13 & 1,36 & 1,81 \\
\hline Outubro & 1,36 & 2,49 & 0,90 & 2,94 & 0,23 & 1,36 \\
\hline Novembro & 0,23 & 3,62 & 1,36 & 1,81 & 0,68 & 0,00 \\
\hline Dezembro & 0,45 & 2,26 & 2,04 & 1,58 & 1,81 & 1,58 \\
\hline \multicolumn{7}{|c|}{ Frequência/sexo } \\
\hline & 2007 & 2008 & 2009 & 2010 & 2011 & 2012 \\
\hline Masculino & 3,39 & 15,38 & 10,41 & 19,46 & 11,76 & 8,60 \\
\hline Feminino & 2,49 & 5,66 & 4,30 & 7,69 & 7,24 & 3,62 \\
\hline \multicolumn{7}{|c|}{ Frequência/ faixa etária } \\
\hline & 2007 & 2008 & 2009 & 2010 & 2011 & 2012 \\
\hline$<1$ & 0 & 0 & 0,23 & 0,45 & 0,45 & 0,23 \\
\hline 1 a 9 & 0,90 & 1,36 & 0,68 & 1,58 & 2,71 & 0,68 \\
\hline 10 a 19 & 1,58 & 4,52 & 3,85 & 5,20 & 2,26 & 3,39 \\
\hline 20 a 34 & 1,36 & 5,43 & 3,85 & 8,60 & 2,49 & 1,81 \\
\hline 35 a 49 & 1,36 & 4,75 & 2,26 & 6,11 & 6,11 & 2,04 \\
\hline$>50$ & 0,68 & 4,98 & 3,85 & 4,98 & 4,98 & 4,07 \\
\hline
\end{tabular}

Fonte: Os Autores.

Observou-se predomínio dos casos da doença nos indivíduos de cor parda $(76,7 \%)$ seguido pelos indivíduos negros (13,35\%), brancos $(4,51 \%)$ e indígenas $(4,08 \%)$ e por fim os indivíduos que se denominaram de raça amarela $(0,23 \%)$ (Tabela 2$)$.

Quanto ao nível de escolaridade, o maior número de casos ocorreu em pessoas que possuíam o ensino fundamental incompleto $(57,24 \%)$. Isso indica que projetos de prevenção e deteç̧ão dos hospedeiros considerados positivos devem ser realizados nas comunidades através de palestras ou ação conjunta com agentes de saúde, para conscientizar a população acerca da doença. 
De acordo com os dados de frequência por zona (Tabela 2), foi observado que a maioria dos casos confirmados estão relacionados a população predominante da zona rural $(61,53 \%)$.

Tabela 2 - Frequência (\%) por raça, ano escolar e zona residencial dos casos de leishmaniose tegumentar americana em indivíduos atendidos nos serviços de saúde do departamento da vigilância à saúde da secretaria de saúde do município de Ilhéus - Bahia, nos anos de $2007-2012$.

\begin{tabular}{|c|c|c|c|c|c|c|}
\hline \multicolumn{7}{|c|}{ Frequência/Raça } \\
\hline & 2007 & 2008 & 2009 & 2010 & 2011 & 2012 \\
\hline Em branco & 0 & 0,45 & 0,00 & 0,23 & 0,00 & 0,45 \\
\hline Branco & 0 & 0,45 & 0,90 & 1,13 & 1,13 & 0,90 \\
\hline Preto & 0,45 & 3,62 & 2,49 & 2,94 & 0,68 & 3,17 \\
\hline Amarelo & 0 & 0 & 0,23 & 0 & 0 & 0 \\
\hline Pardo & 4,98 & 16,29 & 10,41 & 21,49 & 15,84 & 7,69 \\
\hline Indígena & 0,45 & 0,23 & 0,68 & 1,36 & 1,36 & 0 \\
\hline \multicolumn{7}{|c|}{ Frequência/ano escolar } \\
\hline & 2007 & 2008 & 2009 & 2010 & 2011 & 2012 \\
\hline Em branco & 0,68 & 3,84 & 1,58 & 3,16 & 2,94 & 2,71 \\
\hline Analfabeto & 0,23 & 2,94 & 1,58 & 1,81 & 2,04 & 1,58 \\
\hline $\begin{array}{l}\text { Fundamental } \\
\text { incompleto }\end{array}$ & 4,52 & 10,41 & 8,60 & 17,42 & 11,09 & 5,20 \\
\hline $\begin{array}{l}\text { Fundamental } \\
\text { Completo }\end{array}$ & 0,23 & 1,36 & 1,36 & 1,36 & 0,45 & 1,36 \\
\hline Médio incompleto & 0 & 0,45 & 0,68 & 1,13 & 0,23 & 0,45 \\
\hline Médio completo & 0,23 & 1,58 & 0,68 & 1,81 & 1,36 & 0,90 \\
\hline Superior Incompleto & 0 & 0,23 & 0,23 & 0,45 & 0 & 0 \\
\hline Superior Completo & 0 & 0,23 & 0,00 & 0,00 & 0,90 & 0 \\
\hline \multicolumn{7}{|c|}{ Frequência/zona residencial } \\
\hline & 2007 & 2008 & 2009 & 2010 & 2011 & 2012 \\
\hline Em branco & 0 & 0,23 & 0,90 & 2,71 & 1,36 & 0,45 \\
\hline Urbana & 1,36 & 7,47 & 3,62 & 7,47 & 6,33 & 6,56 \\
\hline Rural & 4,52 & 13,35 & 10,18 & 16,97 & 11,31 & 5,20 \\
\hline
\end{tabular}

Fonte: Os Autores.

A tabela 3 demonstra o provável local da fonte de infecção durante os anos de 2007 a 2012, sendo percebido que a maioria dos casos ocorridos foi relatada no município de residência $(85,08 \%)$. Os casos que não ocorreram no município de residência foram de indivíduos contaminados $(11,08 \%)$ e $3,85 \%$ dos casos não sabem onde contraíram a doença.

Através dos dados obtidos na tabela 3 para a frequência das doenças relacionadas ao trabalho, foi possível perceber que a na maioria dos casos a enfermidade confirmada não estava relacionada ao ambiente de trabalho, ou seja, 48,19\% dos indivíduos não contraíram a doença em seu trabalho e $22,84 \%$ dos indivíduos adquiriram durante seu exercício trabalhista, provavelmente em sua maioria em ambientes rurais, próximo a matas e a animais domésticos e silvestres.

Durante essa pesquisa foi feito o levantamento de 442 fichas de pacientes que foram atendidos no município, que residem na área com casos

Tabela 3 - Frequência (\%) por local da fonte de infecção, doença relacionada ao trabalho, classificação epidemiológica e tipo de entrada dos casos de leishmaniose tegumentar americana em indivíduos atendidos nos serviços de saúde do departamento da vigilância à saúde da secretaria de saúde do município de Ilhéus - Bahia, nos anos de 2007 - 2012.

\begin{tabular}{|c|c|c|c|c|c|c|}
\hline \multicolumn{7}{|c|}{ Frequência/autoctone município de residência } \\
\hline & 2007 & 2008 & 2009 & 2010 & 2011 & 2012 \\
\hline Sim & 4,30 & 16,97 & 12,90 & 23,76 & 16,29 & 10,86 \\
\hline Não & 1,58 & 3,62 & 1,58 & 1,58 & 2,04 & 0,68 \\
\hline Indeterminado & 0 & 0,45 & 0,23 & 1,81 & 0,68 & 0,68 \\
\hline \multicolumn{7}{|c|}{ Frequência/doença relacionada ao trabalho } \\
\hline & 2007 & 2008 & 2009 & 2010 & 2011 & 2012 \\
\hline \multirow{2}{*}{$\begin{array}{l}\text { Em branco } \\
\text { Sim }\end{array}$} & 0 & 1,58 & 3,17 & 7,01 & 5,88 & 3,39 \\
\hline & 1,81 & 7,47 & 4,30 & 9,95 & 5,66 & 1,58 \\
\hline Não & 4,07 & 11,99 & 7,24 & 10,18 & 7,47 & 7,24 \\
\hline \multicolumn{7}{|c|}{ Frequência/classificação epidemiológica } \\
\hline & 2007 & 2008 & 2009 & 2010 & 2011 & 2012 \\
\hline Autóctone & 3,62 & 17,19 & 13,12 & 25,34 & 17,87 & 10,41 \\
\hline Importado & 1,81 & 3,39 & 1,58 & 1,36 & 0,90 & 0,68 \\
\hline Indeterminado & 0,45 & 0,45 & 0,00 & 0,45 & 0,23 & 1,13 \\
\hline \multicolumn{7}{|c|}{ Frequência/tipo de entrada } \\
\hline & 2007 & 2008 & 2009 & 2010 & 2011 & 2012 \\
\hline Em Branco & 0 & 0 & 0 & 0 & 0,23 & 0,23 \\
\hline Caso Novo & 5,43 & 21,04 & 14,71 & 26,47 & 18,33 & 11,99 \\
\hline Recidiva & 0,23 & 0,00 & 0,00 & 0,00 & 0,23 & 0 \\
\hline Transferência & 0,23 & 0,00 & 0,00 & 0,68 & 0,23 & 0 \\
\hline
\end{tabular}

Fonte: Os Autores. 
já confirmados de LTA. Segundo classificação epidemiológica do município de Ilhéus, foram confirmados $87,55 \%$ casos são autóctones, $30,77 \%$ são importados e $3,85 \%$ são indeterminados.

Cerca de $100 \%(97,97 \%)$ da frequência de entrada das notificações no setor de vigilância epidemiológica do departamento da vigilância à saúde da secretaria de saúde do município de Ilhéus são de novos casos. O reaparecimento da doença ou do sintoma, após período de cura foi de $0,46 \%$ com apenas dois casos constatados, ou seja, após a cura da doença a possibilidade da mesma retornar se torna mínima.

De acordo com a tabela 4, foi constatado que $61,08 \%$ dos casos foram confirmados laboratorialmente e $38,9 \%$ foram confirmados pelo critério clínico epidemiológico. A forma clínica predominante foi a cutânea $(96,61 \%)$. É importante ressaltar que além de medidas de controle da doença é necessário esclarecer a população sobre os métodos de precaução, transmissão e tratamento por agentes de saúde e população em geral.

Tabela 4 - Frequência (\%) por Critério de confirmação, forma clínica, lesão e cicatrizes dos casos de leishmaniose tegumentar americana em indivíduos atendidos nos serviços de saúde do departamento da vigilância à saúde da secretaria de saúde do município de Ilhéus - Bahia, nos anos de 2007 - 2012.

\begin{tabular}{lcccccc}
\hline \multicolumn{7}{c}{ Frequência/Critério de confirmação } \\
\hline & $\mathbf{2 0 0 7}$ & $\mathbf{2 0 0 8}$ & $\mathbf{2 0 0 9}$ & $\mathbf{2 0 1 0}$ & $\mathbf{2 0 1 1}$ & $\mathbf{2 0 1 2}$ \\
\hline $\begin{array}{l}\text { Clinico } \\
\text { laboratorial }\end{array}$ & 1,36 & 12,44 & 11,99 & 18,55 & 10,86 & 5,88 \\
Clinico Epidêmico & 4,52 & 8,60 & 2,71 & 8,60 & 8,14 & 6,33 \\
\hline \multicolumn{7}{c}{ Frequência/forma clínica } \\
\hline Cutânea & $\mathbf{2 0 0 7}$ & $\mathbf{2 0 0 8}$ & $\mathbf{2 0 0 9}$ & $\mathbf{2 0 1 0}$ & $\mathbf{2 0 1 1}$ & $\mathbf{2 0 1 2}$ \\
\hline Mucosa & 5,20 & 20,81 & 14,48 & 25,57 & 18,33 & 12,22 \\
\hline \multicolumn{7}{c}{ Frequência/lesão cutânea } \\
\hline Sim & 0,68 & 0,23 & 0,23 & 1,58 & 0,68 & 0 \\
\hline Não & $\mathbf{2 0 0 7}$ & $\mathbf{2 0 0 8}$ & $\mathbf{2 0 0 9}$ & $\mathbf{2 0 1 0}$ & $\mathbf{2 0 1 1}$ & $\mathbf{2 0 1 2}$ \\
\hline Não & 5,43 & 21,04 & 14,48 & 26,24 & 19,00 & 12,22 \\
\hline & 0,45 & 0,00 & 0,23 & 0,90 & 0,00 & 0,00 \\
\hline
\end{tabular}

\begin{tabular}{lcccccc}
\hline \multicolumn{7}{c}{ Frequência/cicatrizes cutânea } \\
\hline \multirow{2}{*}{ Em Branco } & $\mathbf{2 0 0 7}$ & $\mathbf{2 0 0 8}$ & $\mathbf{2 0 0 9}$ & $\mathbf{2 0 1 0}$ & $\mathbf{2 0 1 1}$ & $\mathbf{2 0 1 2}$ \\
\multirow{2}{*}{ Sim } & 5,20 & 20,81 & 14,71 & 26,92 & 18,55 & 12,22 \\
Não & 0,45 & 0,00 & 0,00 & 0,00 & 0,45 & 0 \\
\hline
\end{tabular}

Fonte: Os Autores.

De acordo com a tabela 5, constataram-se no ano 2012 casos positivos de LTA em gestantes (tabela 5), responsáveis por $0,23 \%$ de casos registrados. É importante salientar que o uso do antimonial pentavalente não é utilizado em gestantes, pois seu tratamento incide em cuidados locais com observações clínicas e sorológicas. No entanto, casos mais graves cutâneos e de mucosas deve-se debater a probabilidade de tratamento após o sexto mês com doses menores fazendo o controle adequado em laboratórios especializados.

Tabela 5 - Frequência (\%) por gestante dos casos de leishmaniose tegumentar americana em indivíduos atendidos nos serviços de saúde do departamento da vigilância à saúde da secretaria de saúde do município de Ilhéus - Bahia, nos anos de 2007 - 2012.

\begin{tabular}{lcccccc}
\hline \multicolumn{7}{c}{ Frequência/gestante } \\
\hline Em branco & $\mathbf{2 0 0 7}$ & $\mathbf{2 0 0 8}$ & $\mathbf{2 0 0 9}$ & $\mathbf{2 0 1 0}$ & $\mathbf{2 0 1 1}$ & $\mathbf{2 0 1 2}$ \\
Sim & 0 & 0 & 0,23 & 0,23 & 0 & 0 \\
Não & 0 & 0 & 0 & 0 & 0 & 0,23 \\
\hline
\end{tabular}

Fonte: Os Autores.

De 442 pessoas que fizeram a intradermorreação de Montenegro (Tabela 6), 59,04\% foram positivos para leishmaniose, $3,17 \%$ foram negativos e $37,77 \%$ não foram realizados os testes, por falta do reativo ou porque algumas pessoas não compareceram à unidade de referência. Em todos o anos avaliados (20072012), entre as drogas utilizadas nos pacientes que iniciaram o tratamento, a de maior predominância foi a antimonial pentavalente principalmente nos anos de $2008(20,81 \%)$ e $2010(26,92 \%)$ e apenas 1 registro de pentamidina $(0,23 \%), 1$ registro de outras drogas não especificada $(0,23 \%), 1$ registro que não foi utilizada 
nenhuma droga $(0,23 \%)$ e 7 registros em branco $(1,59 \%)$.

Tabela 6 - Frequência (\%) por intradermorreação de Montenegro (IRM), droga administrada e dose prescrita dos casos de leishmaniose tegumentar americana em indivíduos atendidos nos serviços de saúde do departamento da vigilância à saúde da secretaria de saúde do município de Ilhéus - Bahia, nos anos de $2007-2012$.

\begin{tabular}{lcccccc}
\hline \multicolumn{7}{c}{ Frequência/IRM } \\
\hline Positivo & $\mathbf{2 0 0 7}$ & $\mathbf{2 0 0 8}$ & $\mathbf{2 0 0 9}$ & $\mathbf{2 0 1 0}$ & $\mathbf{2 0 1 1}$ & $\mathbf{2 0 1 2}$ \\
Negativo & 1,36 & 11,99 & 11,76 & 18,10 & 10,63 & 5,20 \\
N Realizado & 0 & 1,36 & 1,13 & 0,45 & 0,23 & 0 \\
\hline & 4,52 & 7,69 & 1,81 & 8,60 & 8,14 & 7,01 \\
\hline & Frequência/droga inicial administrada & & \\
\hline Em branco & $\mathbf{2 0 0 7}$ & $\mathbf{2 0 0 8}$ & $\mathbf{2 0 0 9}$ & $\mathbf{2 0 1 0}$ & $\mathbf{2 0 1 1}$ & $\mathbf{2 0 1 2}$ \\
Antimonial & 0,23 & 0,00 & 0,00 & 0,00 & 0,23 & 1,13 \\
Pentavalente & 5,43 & 20,81 & 14,71 & 26,92 & 18,78 & 11,09 \\
Pentamidina & 0,23 & 0 & 0 & 0 & 0 & 0 \\
Outras drogas & 0 & 0,23 & 0 & 0 & 0 & 0 \\
Não Utilizada & 0 & 0 & 0 & 0,23 & 0 & 0 \\
\hline & Frequência/dose prescrita mg/kg/dia & & \\
\hline & $\mathbf{2 0 0 7}$ & $\mathbf{2 0 0 8}$ & $\mathbf{2 0 0 9}$ & $\mathbf{2 0 1 0}$ & $\mathbf{2 0 1 1}$ & $\mathbf{2 0 1 2}$ \\
\hline Em branco & 0,68 & 0,45 & 5,66 & 6,79 & 3,62 & 2,26 \\
$<10$ & 2,26 & 1,81 & 1,58 & 1,36 & 0,45 & 1,13 \\
$\geq 10$ e $<20$ & 2,94 & 18,78 & 4,52 & 19,00 & 14,93 & 8,82 \\
\hline Fo & 0 & 0 & 2,94 & 0 & 0 & 0 \\
\hline
\end{tabular}

Fonte: Os Autores.

A dose de antimonial pentavalente ao dia é prescrita de acordo com o peso do paciente, desse modo, $8,59 \%$ dos pacientes usaram menos que $10 \mathrm{mg} /$ $\mathrm{kg} / \mathrm{dia}, 68,99 \%$ receberam a dose variando entre 10 $20 \mathrm{mg} / \mathrm{kg} / \mathrm{dia}$ e $2,94 \%$ receberam dose $\geq 20 \mathrm{mg} / \mathrm{kg} /$ dia. Esses dados se aproximam dos resultados encontrados por Silva-Nunes et al., (2008) com valores superiores (62\%) utilizados nas doses de $15 \mathrm{mg} / \mathrm{kg} / \mathrm{dia}, 3,6 \%$ com doses de 15 a $20 \mathrm{mg}, 4,9 \%$ superiores a $20 \mathrm{mg}$.

No Brasil, os percentuais de cura e abandono do tratamento em 2007 atingem 77,68\% e 3,74\%, respectivamente (Brasil, 2007). Porém esses indicadores devem ser analisados para a realidade de cada região, estado, município e localidade. Comparado à média nacional, o município de Ilhéus está abaixo dos percentuais de cura com $64,03 \%$ dos casos e com maiores taxas de abandono com $14,26 \%$ (Tabela 7).

Tabela 7 - Frequência (\%) por evolução do caso e óbito por casos de leishmaniose tegumentar americana em indivíduos atendidos nos serviços de saúde do departamento da vigilância à saúde da secretaria de saúde do município de Ilhéus - Bahia, nos anos de $2007-2012$.

\begin{tabular}{lcccccc}
\hline \multicolumn{7}{c}{ Frequência/evolução do caso } \\
\hline & $\mathbf{2 0 0 7}$ & $\mathbf{2 0 0 8}$ & $\mathbf{2 0 0 9}$ & $\mathbf{2 0 1 0}$ & $\mathbf{2 0 1 1}$ & $\mathbf{2 0 1 2}$ \\
\hline Em branco & 0 & 0 & 0,23 & 7,69 & 2,49 & 1,13 \\
Cura & 3,62 & 17,65 & 11,54 & 16,74 & 6,79 & 7,69 \\
Abandono & 0,45 & 1,36 & 0,68 & 0,23 & 9,73 & 1,81 \\
Óbito por outra causa & 0 & 0,23 & 0,00 & 0,00 & 0,00 & 0,23 \\
Transferência & 1,58 & 1,81 & 2,26 & 2,49 & 0,00 & 1,13 \\
Mudança de diagnóstico & 0,23 & 0,00 & 0,00 & 0,00 & 0,00 & 0,23 \\
\hline & Frequência/trimestre do óbito & & & \\
\hline & $\mathbf{2 0 0 7}$ & $\mathbf{2 0 0 8}$ & $\mathbf{2 0 0 9}$ & $\mathbf{2 0 1 0}$ & $\mathbf{2 0 1 1}$ & $\mathbf{2 0 1 2}$ \\
\hline Em branco & 5,88 & 20,81 & 14,71 & 27,15 & 19,00 & 11,99 \\
$1^{\circ}$ trimestre & 0 & 0,23 & 0 & 0 & 0 & 0 \\
$2^{\circ}$ trimestre & 0 & 0 & 0 & 0 & 0 & 0 \\
$3^{\circ}$ trimestre & 0 & 0 & 0 & 0 & 0 & 0 \\
$4^{\circ}$ trimestre & 0 & 0 & 0 & 0 & 0 & 0,23 \\
\hline
\end{tabular}

Fonte: Os Autores.

A frequência de óbito observada (Tabela 7) entre os pacientes investigados no setor de vigilância epidemiológica do departamento da vigilância à saúde da secretaria de saúde do município de Ilhéus - Bahia foram de $0,23 \%$ pacientes durante os anos de 2008 e 2012, porém nenhum desses casos estão relacionados a LTA, e sim, a outras causas.

\section{Discussão}

Os dados encontrados na Tabela 1 são superiores aos achados por Campos Junior (2007) que observou 540 casos em seu estudo analisando a distribuição espacial dos casos humanos de LTA na zona urbana de Ilhéus entre os anos de 2000 e 2004 em apenas 5 anos, ou seja, em 6 anos observados nesse estudo, foram observados resultados inferiores ao estudo supracitado. Rocha et al. (2015) durante o período do estudo, notificaram 547 casos no estado de Alagoas entre os anos de 2007 e 2013, sendo que o ano com 
maior incidência foi o de 2007 com $22,12 \%$ dos casos, diferentemente do presente estudo com $5,88 \%$ das notificações em 2007 denominando o ano de menor ocorrência.

Diante das notificações descritas nas frequências mensais de casos de LTA, constata-se que não há sazonalidade visível durante o ano na ocorrência da doença. De acordo com Lemos e Lima (2005) existe a predominância do vetor em ambiente de clima quente e úmido, declividade, fontes de alimentos e outros parâmetros ecológicos que determinam a distribuição e a possível ocorrência do ciclo da doença.

Reis e Franco (2010) citam que em Manaus as infecções de LTA são frequentes o ano todo, principalmente nos meses em que a precipitação pluviométrica está mais elevada predispondo a população para essa doença, o que é contrario ao presente estudo onde a precipitação não influenciou no número de casos em períodos com maior precipitação.

Os homens foram os mais acometidos pela maior exposição aos fatores de risco, essa incidência provavelmente está relacionada ao seu tipo de atividade ocupacional, ou seja, há maior predominância nas atividades rurais, como agricultura e pecuária (FRANÇA et al., 2009).

A faixa etária encontrada no presente estudo corroboram os resultados encontrados por Oliart Guzmán (2013) e Guerra et al. (2007) que encontraram maiores porcentagens na faixa etária em pessoas com 20 anos de idade avaliando a distribuição por idade dos casos de LTA. Rocha et al. (2015) analisando aspectos epidemiológicos em casos confirmados de LTA encontrou maiores percentuais de casos na faixa etária entre 20-34 anos, no entanto, seus menores valores foram obtidos para os indivíduos com idade acima de 80 anos, contrastando com os resultados obtidos nesse trabalho com menores percentagens em crianças de 1 a 4 anos. A transmissão dessa doença acometida por idosos, mulheres e crianças está relacionada às moradias em locais mais pobres com deficiência de coletas de lixo e abastecimentos de água causando assim um lugar propício à adaptação dos vetores, tal como a convivência com os animais domésticos em suas residências (BENTES et al., 2015).

Segundo Batista et al. (2014) em estudo sobre o perfil epidemiológico no estado do Piauí entre 2007 e 2011, constataram que a maior prevalência da LTA ocorre na fase adulta dos indivíduos na faixa etária entre 20 e 59 anos. De acordo com o Ministério da Saúde (BRASIL, 2007), isto acontece porque os indivíduos nessa faixa etária estão na fase produtiva com encontro ocupacional nas atividades laborais, as quais se relacionam muitas vezes ao desmatamento (entradas em áreas florestais) e atividades militares e ao trabalho doméstico e/ou na lavoura (subsistência), o que demonstra o maior contato com os vetores transmissores que causam essa patologia.

Quanto à raça, Félix et al. (2011) em seu estudo encontraram predomínio da doença sobre indivíduos de cor parda $(66,67 \%)$, seguido por negros $(25,71 \%)$ e brancos $(7,62 \%)$. Esses dados devem ser interpretados a partir dos dados demográficos da população residente por cor, segundo o IBGE (2013) a região do estado da Bahia apresenta em sua maioria uma população de cor parda $(59,2,5 \%)$, seguida pela cor negra $(17,1 \%)$.

Segundo Zhu e Stiller (2002), o perfil do homem do campo é trabalhar em matas e/ou próximo aos animais. Isto torna o trabalhador rural mais suscetível às enfermidades e próximo a locais propícios ao vetor. É perceptivo que o maior número de casos está presente nos indivíduos que trabalham ou residem em zonas rurais, diante disso, de acordo com o presente estudo, observa-se que no ano de 2012 houve inversão das ocorrências referente às zonas, pois o número de casos foi superior para residentes urbanos $(6,56 \%)$, sendo possível afirmar a necessidade de prevenção e controle desses vetores em âmbito urbano diante dos fatos apresentados na tabela 2 .

A forma cutânea da LTA foi a mais encontrada (Tabela 4) corroborando os achados de Naiff Júnior et al. (2009), que avaliando as características clínicas de acordo com os dados analisados de 547 pacientes, demonstraram que houve o predomínio de forma cutânea em 95,06 \% pacientes e em menor proporção de forma cutânea $(4,94 \%)$ com apenas 27 casos.

O resultado encontrado nessa pesquisa está de acordo com resultados encontrados pelo Ministério de Saúde (BRASIL, 2007) que estima que em 3\% a 5\% dos casos de lesão cutânea desenvolvam lesão mucosa. Em casos de presença de lesão mucosa, $98,41 \%$ dos casos encontrados em relação à frequência da LTA por ocorrência de cicatrizes cutâneas foram identificados como ignorados/branco, seguido por $0,9 \%$ dos casos positivos por cicatrizes cutâneas e $0,69 \%$ como sem cicatrizes cutâneas. O grande número de casos em branco se deve a falta de informações essenciais no preenchimento das fichas de notificação dos indivíduos, o que impossibilita a delimitação do perfil do paciente.

A droga de primeira escolha para tratamento é o antimonial pentavalente (Tabela 6), que foi 
padronizado pela Organização Mundial da Saúde na dose entre 10 a $20 \mathrm{mg} / \mathrm{Sb}^{+5} / \mathrm{kg} / \mathrm{dia}$, por 20 a 30 dias. Há drogas alternativas como estibugluconato de pentamidina e anfotericina B, utilizadas nas formas resistentes ao tratamento convencional. No entanto, mesmo o tratamento sendo administrado de forma adequada, a ocorrência de recidivas e/ou comprometimento mucoso é frequente, sendo de $2 \%$ nos casos tratados e em média de $10 \%$ nos casos que não foram tratados (ALMEIDA; SANTOS, 2011). Outras drogas conduzidas de forma tópica ou por via oral têm apresentado resultados encorajadores, muito embora sejam necessários testes clínicos adicionais para se conhecer sua real eficácia. Vale ressaltar o papel do miltefosine, utilizado por via oral em doses de 133 e $150 \mathrm{mg} /$ dia por 4 semanas, que obteve índices de cura da ordem de 100 e $89 \%$, respectivamente (JHA et al., 1999).

Possivelmente, os resultados de frequência por evolução dos casos de LTA (Tabela 7) em indivíduos atendidos nos serviços de saúde sobre as taxas de abandono acima da média nacional, permitem identificar que alguns indivíduos podem ter curado precocemente a lesão sem procurar atendimento médico, o que pode justificar o índice de abandono no presente estudo. Os demais casos são justificados por alguns indivíduos permanecerem meses com a lesão em atividade e o processo de cicatrização mais lenta. Fenômeno este, que pode ser explicado pelo estabelecimento rápido ou tardo de uma resposta imune na eliminação do parasito.

\section{Conclusões}

Observou-se que a partir de 2010 (ano de maior ocorrência) houve um decréscimo significativo diante dos casos positivos para leishmaniose. Os indivíduos que estão mais propícios à doença são do sexo masculino, na faixa etária de 20 a 35 anos, residentes em áreas rurais, da cor parda, autóctone. A forma mais incidente da LTA é a cutânea e a droga mais utilizada para o tratamento é a antimonial pentavalente. Espera-se mostrar a importância da notificação de investigação, diagnóstico precoce e tratamento eficaz para reduzir sequelas, bem como contribuir para a prevenção e controle da doença na cidade.

\section{Referencias}

ALMEIDA, O. L. S.; SANTOS, J. B. Avanços no tratamento da leishmaniose tegumentar do novo mundo nos últimos dez anos: uma revisão sistemática da literatura. Anais Brasileiros de Dermatologia, Rio de Janeiro, v. 86, n. 3, p. 497-506, 2011.

ALVES, C. C. V.; MAGALHÃES, N. A.; SOUSA, G. G. T. Incidência de leishmaniose tegumentar americana no município de Timon/MA. PUBVET: Publicações em Medicina Veterinária e Zootecnia, Londrina, v. 6, n. 30, p. 1- 11, 2012.

BATISTA, F. M. A.; MACHADO, F. F. O. A.; SILVA, J. M. S.; BARJA, J. M. P. R.; SIMIONI, A. R. Leishmaniose: perfil epidemiológico dos casos notificados no estado do Piauí entre 2007 e 2011. Revista Univap, São José dos Campos, v. 20, n. 35, p. 44-55, 2014.

BENTES, A.A.; RODRIGUES, D. E.; CARVALHO, E.; CARVALHO, A. L.; CAMPOS, F. A.; ROMANELLI, R. M. C. Leishmaniose tegumentar americana: um desafio diagnóstico na prática pediátrica. Revista Médica de Minas Gerais, Belo Horizonte, v. 25, n. 6, p. 83-87. 2015.

BRASIL. Ministério da Saúde. Secretaria de Vigilância em Saúde. Departamento de Vigilância Epidemiológica. Manual de Vigilância da leishmaniose tegumentar americana. 2. ed. Brasília: Ministério da Saúde, 2007. 182 p.

CAMPOS JUNIOR, D. A. Distribuição espacial dos casos humanos de leishmaniose tegumentar americana na zona urbana de Ilhéus, Bahia, Brasil, 2000 - 2004. 2007. 68 f. Tese (Doutorado em Medicina Veterinária) - Universidade Federal de Minas Gerais, Escola de Veterinária, Belo Horizonte, 2007.

CARDOSO, R. F.; MELO, B. G.; PEREIRA, W. M. M.; PALÁCIOS, V. R. C. M.; BARBOSA, A. V.; GONÇALVES, N. V. Estudo socioepidemiológico e espacial da leishmaniose tegumentar americana em município do Pará. Revista Paraense de Medicina, Pará, v. 29, n. 3, p. 29-36. 2015. 
FELIX, G. C.; ARAÚJO NETO, F. B.; BACURAU, F. R. S.; BRITO, L. A.; INÁCIO, N. T. D.; SOUSA, P. S. Perfil epidemiológico de pacientes com leishmaniose tegumentar americana no município de Barbalha, CE. Revista de Psicologia, Jaboatão dos Guararapes, v. 1, n. 14, p. 30-35, 2011.

FRANÇA, E. L.; MANDADOR, M. N.; FRANÇA, J. L.; BOtelho, A. C. F.; FERRARI, C. K. B.; FRANÇA, A. C. F. Aspectos epidemiológicos da leishmaniose tegumentar americana no município de Juína, Mato Grosso, Brasil. Scientia Medica, Porto Alegre, v. 19, n. 3, p. 103-107. 2009.

GOMES, J. S.; OLIVEIRA, F. S.; CARDOZO, S. V.; PACHECO, R. S. Importância da técnica de reação em cadeia da polimerase (PCR) no diagnóstico específico de leishmaniose tegumentar americana. Revista UNIABEU, Belford Roxo, v. 8, n. 20, p. 337-349, 2015.

GUERRA, J. A. O.; BARBOSA, M. G. V.; OUREIRO, A. C. S. P.; COELHO, C. P.; ROSA, G. G.; COELHO, L. I. A. C. R. Leishinaniose tegumentar americana em crianças: Aspectos epidemiológicos de casos atendidos em Manaus, Amazonas, Brasil. Cadernos de Saúde Publica, Ro de Janeiro, v. 23, n. 9, p. 2215 2223, 2007.

IBGE. Pesquisa Nacional por Amostra de Domicilio (PNAD). 2013. Disponível em: $<$ http://www.ibge.gov. br>. Acesso em: 12 nov. 2016.

IBGE. Cidades. 2010. Disponível em: < http://www. ibge.gov.br>. Acesso em: 12 nov. 2016.

JHA, T. K.; SUNDAR, S.; THAKUR, C. P.; BACHMANN, P.; KARBWANG, J.; FISCHER, C.; VOSS, A.; BERMAN, J. Miltefosine, an oral agent, for the treatment of Indian visceral leishmaniosis. The New England Journal of Medicine, Boston, v. 341, n. 24, p. 1795-1800, 1999.

LEMOS, J. C.; LIMA, S. C. Leishmaniose tegumentar americana: Flebotomínios em áreas de transmissão no município de Uberlândia-MG. Revista da Sociedade Brasileira de Medicina Tropical, Brasília, v. 38, n. 1, p. 22-26, 2005.
MOTA, L. A. A.; MIRANDA, R. R. Manifestações dermatológicas e otorrinolaringológicas na Leishmaniose. Arquivos Internacionais de Otorrinolaringologia. São Paulo, v. 15, n. 3, p. 376381, 2011.

MOURA, I. M. Epidemiologia da leishmaniose tegumentar americana: uma revisão sistemática. 2013. 35 f. Monografia (Trabalho de Conclusão de Curso) - Universidade Federal da Bahia, Faculdade de Medicina da Bahia, Salvador, 2013.

NAIFF JÚNIOR, R. D.; PINHEIRO, F. G.; NAIFF, M. F.; SOUZA, I. S.; CASTRO, L. M.; MENEZES, M. P.; FRANCO, A. M. R. Estudo de uma série de casos de leishmaniose tegumentar americana no município de Rio Preto da Eva, Amazonas, Brasil. Revista de Patologia Tropical, Goiânia, v. 38, n. 2, p. 103-14, 2009.

NOBRES, E. S.; SOUZA, L. A.; RODRIGUES, D. J. Incidência de leishmaniose tegumentar americana no norte de Mato Grosso entre 2001 e 2008. Acta Amazônica, Manaus, v. 43, n. 3, p. 297-304, 2013.

OMS. Organização Mundial de Saúde. The Leishmaniasis and Leishmania/HIV co-infections. 2000. Disponível em: <http:/www.who.int/inf-fs/em/ fact116.html>. Acesso em: 26 ago. 2016.

OLIART-GUZMÁN, H.; MARTINS, A. C.; MANTOVANI, S. A. S.; BRAÑA, A. M.; DELFINO, B. M.; PEREIRA, T. M.; SANTOS, A. P.; FILGUEIRA JÚNIOR, J. A.; CASTELO BRANCO, F. L. C.; CAMPOS, R. G.; OLIVEIRA, C. S. M.; MUNIZ, P. T.; SILVA-NUNES, M. Caracteristicas epidemiológicas da leishimaniose tegumentar americana na fronteira amazônica: estudo retrospectivo em Assis Brasil, Acre. Revista de Patologia Tropical, Goiânia, v. 42, p. 187-200, 2013.

REIS, S. R.; FRANCO, A. M. R. A Leishmaniose tegumentar americana no estaddo do Amazonas, Brasil. Aspectos epidemológicos da leishmaniose canina. Revista CFMV, Brasilia, v. 50, n. 50, p. 35-40, 2010. 
ROCHA, T. J.; SANTANA, E. P. C.; BARBOSA, A. C. A.; CALHEIROS, C. M. L. Aspectos epidemiológicos dos casos de leishmaniose tegumentar americana. Revista Pan-Amazônica de Saúde, Ananindeua, v. 6, n. 4, p. 49-54, 2015.

SANTANA, A. F.; SANTANA, F. F.; SANTANA, L. F.; REIS, D. J. S.; NEVES, S. J. Uma breve história econômica de Ilhéus: gênese, apogeu e declínio da lavoura cacaueira. In: SEMANA DO ECONOMISTA, 4., ENCONTRO DE EGRESSOS, 4., 2014, Ilhéus. . Anais... Ilhéus: Universidade Estadual de Santa Cruz, 2014.

SILVA-NUNES, M.; CAVASINI, C. E.; SILVA, N. S.; GALATI, E. A. B. Epidemiologia da leishmaniose tegumentar e descrição das populações de flebotomíneos no município de Acrelândia, Acre, Brasil. Revista Brasileira de Epidemiologia, São Paulo, v. 11, n. 2, p. 241-251, 2008.

ZHU, Y. I.; STILLER, M. J. Arthropods and skin diseases. International Journal of Dermatology, Washington, v. 41, n. 9, p. 533-49, 2002. 